\title{
Comparative analysis of leaf photosynthetic characteristics and fruit sugar content in trees of Citrus cultivar 'Huangguogan' of different age
}

\author{
B. XIONG ${ }^{*+}$, X. QIU*, S.J. HUANG ${ }^{*}$, Y.T. YANG*, G.C. SUN ${ }^{* *}$, X.J. WANG ${ }^{*}$, X. ZHANG ${ }^{*}$, \\ T.T. DONG ${ }^{*}$, X.Y. SHEN ${ }^{*}$, W. WEI ${ }^{*}$, and Z.H. WANG ${ }^{*, * *,+}$ \\ College of Horticulture, Sichuan Agricultural University, 611130 Chengdu, China* \\ Institute of Pomology and Olericulture, Sichuan Agricultural University, 611130 Chengdu, China**
}

\begin{abstract}
Citrus trees of cv. Huangguogan, 8-, 30-, and 280-year-old (8s, 30s, and 280s, respectively), were used to explore the photosynthesis and related enzyme activity. In spring shoot leaves, net photosynthetic rate $\left(P_{\mathrm{N}}\right)$ of the 280 s was significantly lower than that of the $30 \mathrm{~s}$ and $8 \mathrm{~s}$, but it was opposite in summer shoot leaves. $P_{\mathrm{N}}$ of the summer shoots was higher than that of the spring shoots. $P_{\mathrm{N}}$ was significantly positively correlated with stomatal conductance, intercellular $\mathrm{CO}_{2}$ concentration, and transpiration rate, but was negatively correlated with maximum photochemical quantum yield of PSII photochemistry. The effect of age on variable fluorescence/minimal fluorescence was similar to that of minimal fluorescence, variable fluorescence, maximal fluorescence yield of the dark-adapted state. Fruits of the 280s had significantly lower sugar components than those of the $8 \mathrm{~s}$ and $30 \mathrm{~s}$. Results showed that fruits of the $8 \mathrm{~s}$ and $30 \mathrm{~s}$ actively accumulated photosynthesis products, which was verified by the higher $P_{\mathrm{N}}$ in the spring shoots, lower acidic invertase and neutral invertase activities, and higher sucrose phosphate synthase than that of the $280 \mathrm{~s}$.
\end{abstract}

Additional key words: chlorophyll fluorescence; flavor; sugar component.

\section{Introduction}

Citrus is a commercially important genus of the family Rutaceae and is widely cultivated for its fruit worldwide (Biswas et al. 2011). Naturally occurring and cultivated hybrids include commercially important fruits, such as the oranges, grapefruit, lemons, some limes, and some tangerines. Hybrid 'Huangguogan' (Citrus reticulata $\times$ Citrus sinensis) has more than 300 years of cultivation history in Shimian County of Sichuan Province, China (Xiong et al. 2017). In recent years, the planting area of 'Huangguogan' has expanded rapidly in southwestern China, but the quality of 'Huangguogan' fruits of different ages differs.

The flavor and quality of fruits are mainly determined by sugars, organic acids, ripeness, amino acids, and aromatic compounds (Zhang et al. 2012). Starch and sugars are the main end products of photosynthesis, and their contents in plants provide key information related to plant productivity. The partitioning between starch and sugar is regulated in the cell in response to various stimuli inside and outside the cell (Rosa et al. 2009). The physiological mechanisms controlling biomass allocation are important for plant growth performance (Poorter et al. 2012). Sugars, especially sucrose, are used by the plant as a carbon source for supporting growth, development, reproduction, and metabolism (Gibson 2005, Basson et al. 2010) and as a signal molecule to regulate the expression of genes related to photosynthesis and heterotrophic metabolism (Koch 1996, Roitsch 1999). In most of the higher plants, sugar acts similarly to hormones as it helps transition from the plants' nutritional state to regulate growth and floral transition. Soluble sugars in leaf and root, particularly sucrose and hexoses (glucose and fructose), are highly sensitive to environmental stresses, and act as cellular respiratory substrates and osmotic regulators to maintain cellular homeostasis (Roitsch and González 2004, Gupta and Kaur 2005, Mishra and Dubey 2008, Maestri et al. 2010, Ruan et al. 2010). However, the plant cannot utilize sucrose directly - sucrose must be first cleaved into hexoses by invertase (EC 3.2.1.26) (Zhang et al. 2014) or sucrose synthase (SS, EC 2.4.1.13) (Koch 2004, Roitsch

\footnotetext{
Received 21 June 2019, accepted 15 May 2020.

${ }^{+}$Corresponding author; phone/fax: 028-8629 1848, email: wangzhihui318@126.com, xiongbo1221@sicau.edu.cn

Abbreviations: AI - acidic invertase; A/N-INV - alkaline/neutral invertase; Chl - chlorophyll; $C_{\mathrm{i}}-$ intercellular $\mathrm{CO}_{2}$ concentration; $E$ - transpiration rate; $\mathrm{ETR}$ - electron transport rate; $\mathrm{F}_{0}$ - minimal fluorescence yield of the dark-adapted state; $\mathrm{F}_{0}{ }^{\prime}$ - minimal fluorescence yield of the light-adapted state; $\mathrm{F}_{\mathrm{m}}$ - maximal fluorescence yield of the dark-adapted state; $\mathrm{F}_{\mathrm{m}}{ }^{\prime}$ - maximal fluorescence yield of the lightadapted state; $F_{s}$ - steady-state fluorescence yield; $F_{v} / F_{m}-$ maximum photochemical quantum yield of PSII photochemistry; $F_{v}{ }^{\prime} / F_{m}{ }^{\prime}-$ actual photochemical efficiency; $g_{\mathrm{s}}$ - stomatal conductance; HK - hexokinase; INV - invertase; NPQ - nonphotochemical quenching; $P_{\mathrm{N}}$ - photosynthetic rate; PPP - pentose phosphate pathway; $\mathrm{q}_{\mathrm{P}}$ - photochemical quenching coefficient; ROS - reactive oxygen species; SPS - sucrose phosphate synthase; SS - sucrose synthase; $\Phi_{\mathrm{PSII}}$ - effective quantum yield of PSII.

Acknowledgements: This work was supported by the Foundation of the Science and Technology Department of Sichuan Province, China (2019NZZJ0013, 2016NZ0056).
} 
and González 2004). Invertases produce glucose, while sucrose synthase produces uridine diphosphate (UDP) glucose; thus, invertases form more hexoses and are thought to have a better ability to regulate gene expression and stimulate specific sugar sensors (Koch 2004).

Among the enzymes involved in sucrose metabolism, $\mathrm{SS}$ and invertase (INV, EC 3.2.1.26) are involved in the cleavage of sucrose, while sucrose phosphate synthase (SPS, EC 2.4.1.14) catalyzes the synthesis of sucrose (Guo et al. 2002). INVs are important for carbohydrate supply to sink tissues; they play a key role in regulating, amplifying, and integrating different signals, which leads to the transportation of end product of photosynthesis from source to sink (Koch 2004, Roitsch and González 2004). There are two main groups of INVs in plants, namely acidic invertase (AI) and alkaline/neutral invertase (A/N-INV) (Dahro et al. 2016). AI maintains tolerance mechanisms to osmotic stress (Wang et al. 2000), salinity (Balibrea et al. 2003), heat ( $\mathrm{Li}$ et al. 2012), heavy metals (Huang et al. 2008, 2011; Xiong et al. 2008), and pathogen invasion (Sturm and Tang 1999). Maintaining carbohydrate balance and primary metabolic pathways is the basis for stress resistance in plants (Stobrawa and Lorenc-Plucińska 2007). The close relationship between $\mathrm{A} / \mathrm{N}-\mathrm{INV}$ and reducing sugar content is assumed to regulate and control photosynthesis and carbon metabolism in chloroplasts, respectively (Lou et al. 2007, Vargas et al. 2008, Lobo et al. 2015), and thus supply some energy products to repair injury from abiotic and biotic stress (Vargas et al. 2007, Martín et al. 2013). In addition, INVs are considered to be closely related to hexokinase (HK), which provides phosphorylates glucose to regulate ROS balance (Foyer and Shigeoka 2011, Xiang et al. 2011, Keunen et al. 2013). In chloroplasts, hexokinase-derived glucose-6phosphate participates in the oxidative pentose phosphate pathway (PPP), an important mechanism to control the removal of $\mathrm{H}_{2} \mathrm{O}_{2}$ (Zeeman et al. 2004, Giese et al. 2005). Compared with other photosynthetic enzymes, ribulose1,5-bisphosphate carboxylase/oxygenase (Rubisco) is considered to be inefficient, which may be due to its low saturation, low $\mathrm{CO}_{2}$ fixation rate, and low affinity for $\mathrm{CO}_{2}$ (Young et al. 2016). Therefore, it is generally believed that photosynthetic enzymes serve important functions in determining fruit quality, especially in terms of sucrose metabolism.

The nature and concentration of sugar, organic acid, cellulose, and secondary metabolites are dynamically changed during fruit development, and largely affect taste characteristics and organoleptic quality (Patel et al. 2015, Dong et al. 2019). Sugar, which is mainly in the form of sucrose, fructose, and glucose, plays an key role in the quality and flavor, and the differences in sugar content determine the sweetness and color of fruit (Dai et al. 2016). Previous research has found that xylose and arabinose were the main sugar component in early fruit development, whereas fructose, glucose, and sucrose showed a significant increase during fruit maturation (Deng et al. 2019). The ripening process of citrus fruits is usually accompanied by the accumulation of sugars and the degradation of organic acids (Liu et al. 2007). Stomatal closure, lower chlorophyll
(Chl) content and altered Rubisco activity are considered the main limiting factors for $P_{\mathrm{N}}$, carbohydrate synthesis, and consequent sugar accumulation (Ye et al. 2019).

The importance of sucrose metabolism in the development of plants has been studied with regard to the pathway of sugar signaling (Koch 2004). However, functional analysis of the six enzymes in the current study has been limited and studies regarding the effects of different ages on the photosynthetic characteristics of citrus trees are scarce. Therefore, we sought to understand differences in photosynthesis and its end products and related enzyme activities in 'Huangguogan' trees of various ages. We examined quantitative changes in important carbohydrates through measures of photosynthetic activity and Chl fluorescence and related enzymes in leaves of 'Huangguogan' trees of different ages during fruit growth as well as sugar contents in ripe fruits. Here, we present our analysis of the relationship between the net photosynthetic rate $\left(P_{\mathrm{N}}\right)$ and other measures of photosynthetic gas-exchange parameters in 'Huangguogan' leaves. This study aims to examine the effects of different tree ages on the sugar accumulation mechanism of citrus and provide a scientific basis for further study on photosynthetic physiology of citrus trees of different age.

\section{Materials and methods}

Plant materials: All the field-grown 'Huangguogan' trees were cultivated in the National Agricultural Science and Technology Park of Ya'an, Sichuan Province, China. The canopy size, tree vigor of different ages 'Huangguogan' trees was basically the same. Stands of 8-year-old (8s), 30-year-old (30s), and 280-year-old (280s) 'Huangguogan' trees were maintained under the same conditions (i.e., fertilization, soil management, disease control, and pruning) as described by Liao et al. (2016) more than five years. Each group had four replicates, so the total number of the experimental 'Huangguogan' plants was 12 (3 groups $\times$ 4 replicates), all the planting spacing was $4 \times 5 \mathrm{~m}$.

Gas exchange and Chl fluorescence measurements: Net photosynthetic rate $\left(P_{\mathrm{N}}\right)$, transpiration rate $(E)$, intercellular $\mathrm{CO}_{2}$ concentration $\left(C_{\mathrm{i}}\right)$, stomatal conductance $\left(g_{\mathrm{s}}\right)$, and the $\mathrm{Chl}$ fluorescence were monitored by portable photosynthesis measuring instrument (LI-6400, Licor, Lincoln, NE, USA). The photosynthetic active radiation and $\mathrm{CO}_{2}$ concentration were $1,200 \mu \mathrm{mol}$ (photon) $\mathrm{m}^{-2} \mathrm{~s}^{-1}$ and $400 \mu \mathrm{mol} \mathrm{mol}{ }^{-1}$, respectively. $g_{\mathrm{s}}$ and $C_{\mathrm{i}}$ were determined at a saturated light intensity of $1,000 \mu \mathrm{mol}$ (photon) $\mathrm{m}^{-2} \mathrm{~s}^{-1}$, and $70 \%$ relative humidity. On the periphery and middle of the tree canopy (about $1.8 \mathrm{~m}$ above the ground), the $4^{\text {th }}$ leaves from top to bottom on the spring and summer shoots of the current year were selected randomly for each replicate during the fruit-expanding period (10 September 2018). The following Chl fluorescence parameters were measured using a LI-6400 infrared gas analyzer (Licor, Lincoln, NE, USA), i.e., minimal fluorescence yield of the dark-adapted state $\left(\mathrm{F}_{0}\right)$, maximal fluorescence yield of the dark-adapted state $\left(\mathrm{F}_{\mathrm{m}}\right)$, minimal fluorescence yield of the light-adapted state $\left(\mathrm{F}_{0}{ }^{\prime}\right)$, 
maximal fluorescence yield of the light-adapted state $\left(F_{m}{ }^{\prime}\right)$, steady-state fluorescence yield $\left(F_{s}\right)$, and electron transport rate (ETR). The maximal quantum yield of PSII, $F_{\mathrm{v}} / F_{\mathrm{m}}=\left(\mathrm{F}_{\mathrm{m}}-\mathrm{F}_{0}\right) / \mathrm{F}_{\mathrm{m}}$, effective quantum yield, $\Phi_{\mathrm{PSII}}=$ $\left(\mathrm{F}_{\mathrm{m}}{ }^{\prime}-\mathrm{F}_{\mathrm{s}}\right) / \mathrm{F}_{\mathrm{m}}{ }^{\prime}$, photochemical quenching coefficient, $\mathrm{q}_{\mathrm{p}}=$ $\left(\mathrm{F}_{\mathrm{m}}{ }^{\prime}-\mathrm{F}_{\mathrm{s}}\right) /\left(\mathrm{F}_{\mathrm{m}}{ }^{\prime}-\mathrm{F}_{0}{ }^{\prime}\right)$, and nonphotochemical quenching, $\mathrm{NPQ}=\left(\mathrm{F}_{\mathrm{m}}-\mathrm{F}_{\mathrm{m}}{ }^{\prime}\right) / \mathrm{F}_{\mathrm{m}}{ }^{\prime}$ were calculated according to the methods of Xie et al. (2018).

Enzyme extraction and activity analysis: In total, 24 samples of the $4^{\text {th }}$ to $6^{\text {th }}$ leaf from top to bottom of currentyear spring and summer shoots were selected randomly from each replicate at 9:00 h, immediately frozen in liquid nitrogen, and stored at $-80^{\circ} \mathrm{C}$ until analysis. Photosynthetic metabolizing enzymes were prepared from frozen tissues as described by Zhang et al. (2011b). AI, NI, SPS, SS, Rubisco, and HK activities were assayed using the Plant AI, NI, SPS, SS, Rubisco, and HK ELISA test kits (Shanghai BOYE Biology Science and Technology Co. Ltd., Shanghai, China) according to the manufacturer's instructions.

Sugar composition: In total, 24 fruit samples were randomly selected from each replicate at 9:00 h when fruits were ripe (25 March 2016), immediately frozen in liquid nitrogen, and stored at $-80^{\circ} \mathrm{C}$ until analysis. Glucose, fructose, and sucrose contents were analyzed using high performance liquid chromatography ( $L C-1260$, Agilent Technologies, Sacramento, CA, USA) according to the methods of Zhang et al. (2012). Samples were isolated with Innoval $\mathrm{NH}_{2}$ column $(4.6 \times 250 \mathrm{~mm}, 5 \mu \mathrm{m}$, Agela Technologies, Tianjin, China) at room temperature. The HPLC experiment was completed using a mobile phase [acetonitrile:water $=80: 20, \mathrm{v} / \mathrm{v}$ ], $20-\mu \mathrm{L}$ sample volume, flow rate of $1 \mathrm{~mL} \mathrm{~min}^{-1}$, column temperature of $30^{\circ} \mathrm{C}$, and detection temperature of $40^{\circ} \mathrm{C}$.

Statistical analysis: All experiments were performed in triplicate $(n=3)$. The data were analyzed using Duncan's multiple range test in the XLSTAT program (version 2010) at the $P<0.05$ level of significance.

\section{Results}

Photosynthesis and Chl fluorescence: The $P_{\mathrm{N}}, g_{\mathrm{s}}, C_{\mathrm{i}}$, and $E$ of leaves taken from the spring shoots of the 280 s was significantly lower than those from the $30 \mathrm{~s}$ and $8 \mathrm{~s}$, respectively (Fig. 1), but the opposite trend was observed in the summer shoots. There was no significant difference in $P_{\mathrm{N}}, g_{\mathrm{s}}, C_{\mathrm{i}}$, and $E$ between $30 \mathrm{~s}$ and $8 \mathrm{~s}$ both in spring and summer shoots.

Regarding the indicators of Chl fluorescence, $\mathrm{F}_{0}, \mathrm{~F}_{\mathrm{m}}$, and $\mathrm{F}_{\mathrm{v}}$ in the leaves of the 280s from both spring and summer shoots were significantly higher than those of the 30 s and 8s (Fig. 2A-C). There were no significant differences between leaves of different ages of 'Huangguogan' trees in terms of $\mathrm{F}_{\mathrm{v}} / \mathrm{F}_{\mathrm{m}}$ and $\mathrm{F}_{\mathrm{v}}{ }^{\prime} \mathrm{F}_{\mathrm{m}}{ }^{\prime}$ (Fig. $2 D, F$ ). The effect of age on $F_{v} / F_{0}$ was similar to trends seen in $F_{0}, F_{v}$, and $F_{m}$ individually; specifically, $\mathrm{F}_{\mathrm{v}} / \mathrm{F}_{0}$ was significantly higher in the leaves of the 280s than those of other ages (Fig. $2 E$ ).

The relationships between $P_{\mathrm{N}}$ and $g_{\mathrm{s}}, C_{\mathrm{i}}, E$, and $\mathrm{F}_{\mathrm{v}} / \mathrm{F}_{\mathrm{m}}$ are demonstrated in Fig. 3. $P_{\mathrm{N}}$ was positively and significantly correlated with $g_{\mathrm{s}}\left(R^{2}=0.8839\right), C_{\mathrm{i}}\left(R^{2}=0.4249\right)$, and $E\left(R^{2}=0.9414\right)$, but negatively correlated with $\mathrm{F}_{\mathrm{v}} / \mathrm{F}_{\mathrm{m}}$ $\left(R^{2}=0.0677\right)$. Our results indicate that $g_{\mathrm{s}}, C_{\mathrm{i}}, E$, and $\mathrm{F}_{\mathrm{v}} / \mathrm{F}_{\mathrm{m}}$ accounted for $88.4,42.5,94.1$, and $6.8 \%$, respectively, of the change in $P_{\mathrm{N}}$.

Photosynthesis-related enzyme activities: The activities of AI, neutral invertase, SS, SPS, Rubisco, and HK in leaves were all significantly related to the tree age (Fig. 4). The AI, NI, and HK activities of leaves from the spring shoots were higher than those from the summer shoots, but SPS and Rubisco showed the opposite trend. Interestingly, the SS activity of leaves from the spring shoots was higher than that of leaves from the summer shoots in the 280s, but this trend was reversed in the 30 s and 8 s.

Sugar components: In the current study, we measured fructose, glucose, and sucrose contents of 'Huangguogan'

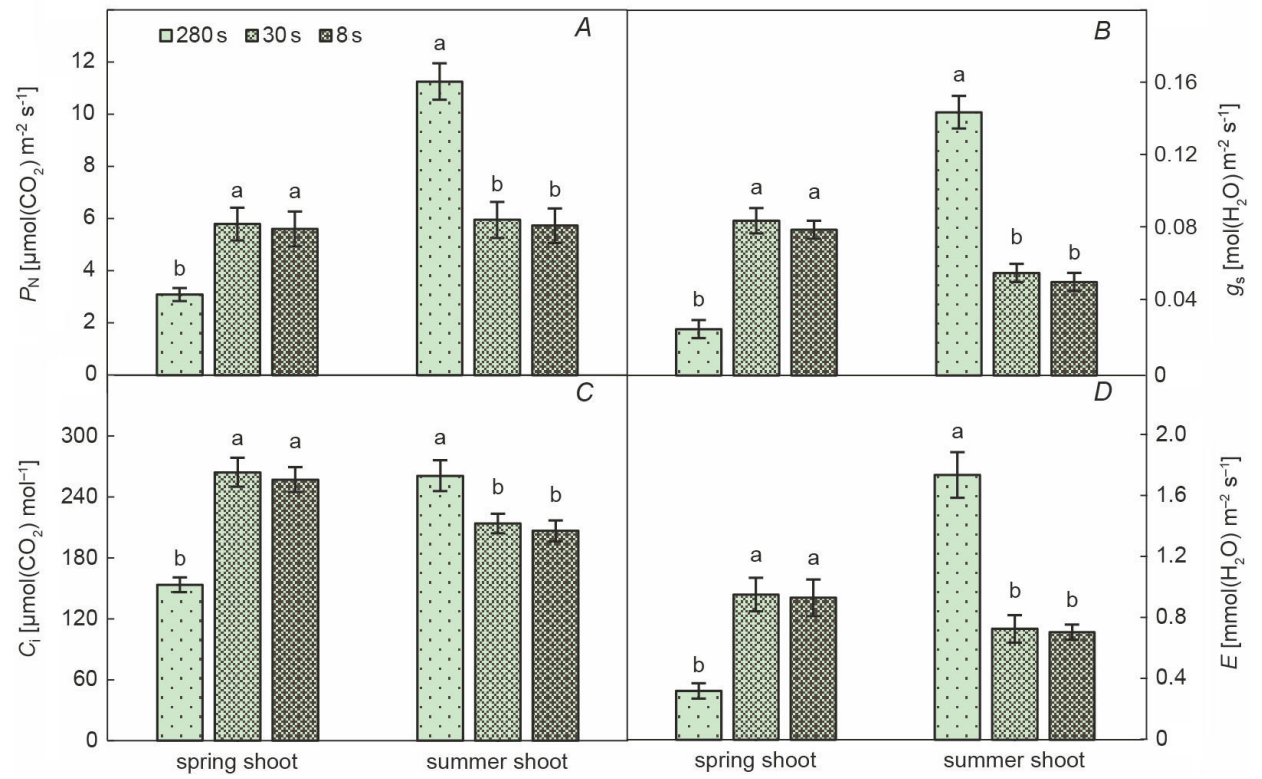

Fig. 1. Changes in the net photosynthetic rate, $P_{\mathrm{N}}(A)$, stomatal conductance, $g_{\mathrm{s}}(B)$, intercellular $\mathrm{CO}_{2}$ concentration, $C_{\mathrm{i}}(C)$, and transpiration rate, $E(D)$ in the leaves of 'Huangguogan' trees of different ages. Values in each column with different letters are significantly different at $P<0.05$. Data are provided as mean $\pm \mathrm{SD}$ of three independent measurements. 


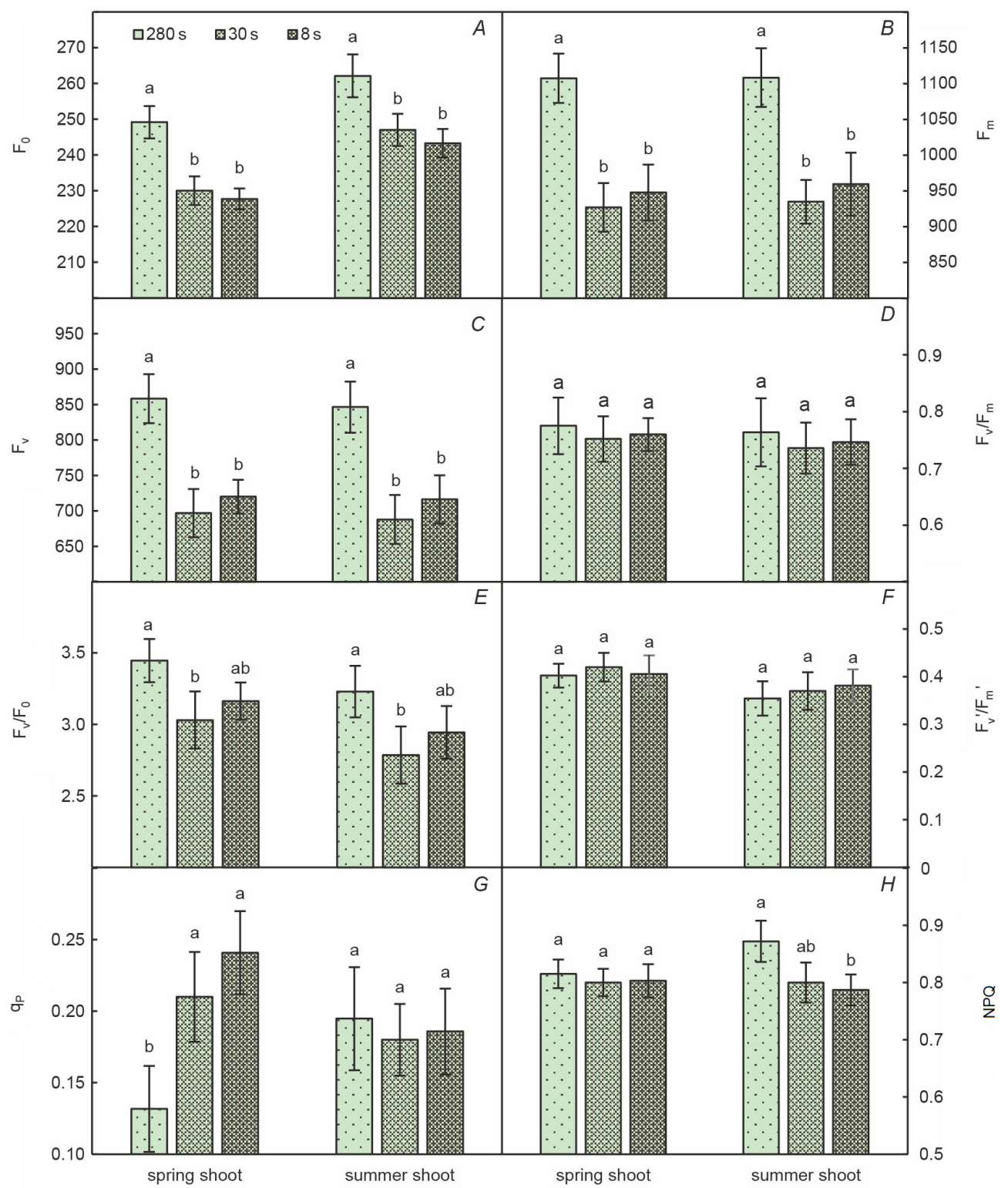

Fig. 2. Chlorophyll fluorescence characteristics of the leaves of 'Huangguogan' trees of different ages. (A) $\mathrm{F}_{0}$, minimum chlorophyll fluorescence yield obtained from dark-adapted leaves. $(B) \mathrm{F}_{\mathrm{m}}$, maximum chlorophyll fluorescence yield obtained from dark-adapted leaves. (C) $\mathrm{F}_{\mathrm{v}}$, variable fluorescence of the dark-adapted samples. $(D) \mathrm{F}_{\mathrm{v}} / \mathrm{F}_{\mathrm{m}}$, maximal photochemical efficiency of PSII in the dark. $(E) \mathrm{F}_{\mathrm{v}} / \mathrm{F}_{0}$, potential activity of PSII. $(F) \mathrm{F}_{\mathrm{v}}{ }^{\prime} / \mathrm{F}_{\mathrm{m}}$ ', photochemical efficiency of PSII in light. $(G)$ q , photochemical quenching. $(H) \mathrm{NPQ}$, nonphotochemical quenching. Different letters above the bars indicate significant differences at the 5\% probability level.

fruits when fruit was ripe. Sucrose was the predominant sugar in ripe 'Huangguogan' fruits from the $8 \mathrm{~s}$ and $30 \mathrm{~s}$, but not the $280 \mathrm{~s}$. Compared to fruits from the $30 \mathrm{~s}$ and $8 \mathrm{~s}$, fructose, glucose, and sucrose contents were all significantly lower in fruits from the 280 s. There was no significant difference between the fruits from the 30 s and 8 s (Fig. 5A-C). Diametrically, the sucrose/hexose ratio of 280 s was lower than that of $30 \mathrm{~s}$ and $8 \mathrm{~s}$, but had no significant effect on sucrose/hexose ratio (Fig. 5D).

\section{Discussion}

In the current study, there were major differences in
'Huangguogan' fruit quality with the increasing tree age, especially with regard to sugar contents. Several physiological characteristics and processes may affect $P_{\mathrm{N}}$, i.e., light, temperature, water quality, soil, and abiotic stresses (Marsic et al. 2018, Ohnishi et al. 2019, Jahan et al. 2020), and these factors can interact to limit photosynthesis and to increase photooxidative damage in sensitive plants (Paudel et al. 2016). The accumulation of photosynthetic products is the result of $g_{\mathrm{s}}, C_{\mathrm{i}}, E$, and chlorophyll fluorescence (Song et al. 2011). $P_{\mathrm{N}}$ is the most representative photosynthetic characteristic of plants, and its value directly reflects photosynthetic capacity (Sicher and Bunce 2001). $C_{\mathrm{i}}$ is an important indicator of stomatal activity. In photosynthesis, 

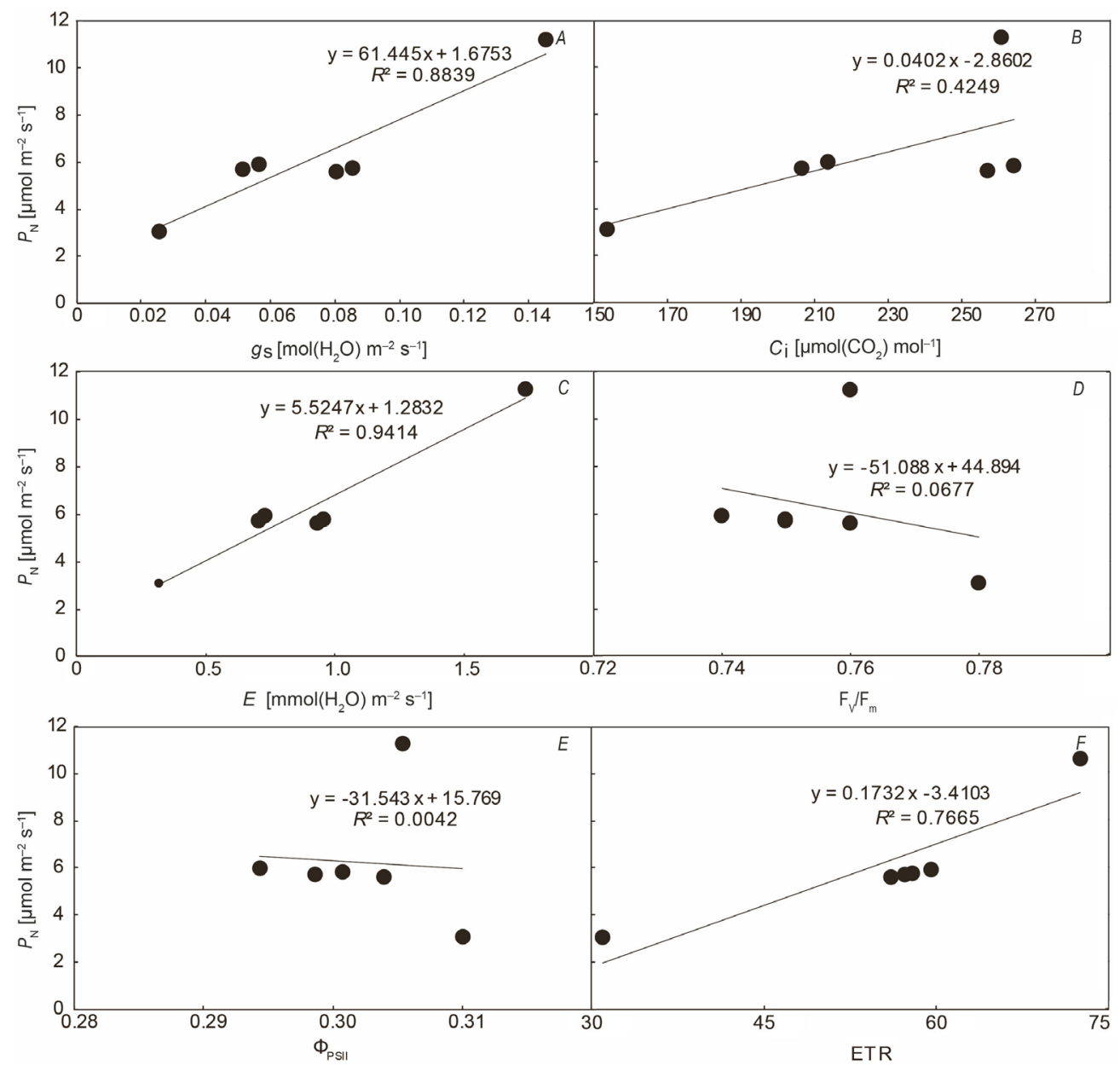

Fig. 3. Linear correlation between net photosynthetic rate $\left(P_{\mathrm{N}}\right)$ and stomatal conductance, $g_{\mathrm{s}}(A)$; intercellular $\mathrm{CO}_{2}$ concentration, $C_{\mathrm{i}}(B)$; and transpiration rate, $E(C)$; maximal photochemical efficiency of PSII in the dark, $\mathrm{F}_{\mathrm{v}} / \mathrm{F}_{\mathrm{m}}(D)$; effective quantum yield of PSII, $\Phi_{\mathrm{PSII}}(E)$; electron transport rate, ETR $(F)$.

a decrease in $E$ caused by a decrease in $g_{\text {s }}$ leads to a significant decrease in transpiration, which can slow down the absorption and transportation of water and nutrients (Gao et al. 2016). In the present study, the $P_{\mathrm{N}}, g_{\mathrm{s}}, C_{\mathrm{i}}$, and $E$ values of leaves from the summer and spring shoots of 'Huangguogan' trees of different ages showed the same patterns of variation. The $P_{\mathrm{N}}$ of leaves from the $280 \mathrm{~s}$ were significantly lower than those of the $30 \mathrm{~s}$ and $8 \mathrm{~s}$ in the spring shoots, indicating that stomatal closure and altered Rubisco activity are probably the main limiting factor for $P_{\mathrm{N}}$. Lower $P_{\mathrm{N}}$ led to less sugar accumulation with increasing tree age (Figs. 1, 4). This phenomenon is consistent with previous research results (Yousuf et al. 2017, Ye et al. 2019). However, the trend was reversed in the summer shoots (Fig. 1), and there were no significant differences in leaves from the spring and summer shoots taken from the $30 \mathrm{~s}$ and $8 \mathrm{~s}$. Among ripe fruits, the fructose, glucose, and sucrose contents in the 280 s were the lowest, and these values were significantly different from those of the $30 \mathrm{~s}$ and 8s (Fig. 5). These results indicated that leaves of the spring shoots might be the main organ for the accumulation of photosynthetic products in 'Huangguogan'.
Chl fluorescence reflects the changes in thylakoid membrane function and organization, as well as the inhibition of photosynthesis and oxygen evolution through interactions with PSII components (Hussain and Reigosa 2011). $F_{0}$ reflects fluorescence when the reaction centers of PSII are all open, and an increase in $\mathrm{F}_{0}$ indicates initial phase of injury to PSII (Kitajima and Butler 1975). $F_{0}$ was significantly higher in the 280 s than that in the other two age groups. It is possible that increased $\mathrm{F}_{0}$ values were due to the disassociation of LHCII and the PSII reaction center in swollen thylakoids (Tsai et al. 2019). $\mathrm{F}_{\mathrm{v}} / \mathrm{F}_{\mathrm{m}}$ represents the conversion efficiency of primary light energy (Guidi et al. 2007) and the potential maximum photosynthetic capacity (Zhou et al. 2016). The effect of different tree ages on $\mathrm{F}_{\mathrm{v}} / \mathrm{F}_{0}$ was similar to that on $\mathrm{F}_{0}, \mathrm{~F}_{\mathrm{v}}$, and $\mathrm{F}_{\mathrm{m}} . \mathrm{F}_{\mathrm{v}} / \mathrm{F}_{0}$ was significantly higher in the 280 s than that in the other two groups (Fig. 2E). In contrast, previous studies have found that there is a strong correlation between $F_{v} / F_{m}$ and $F_{v} / F_{0}$ (Sharma et al. 2014). The result of this study indicated that $\mathrm{F}_{\mathrm{v}} / \mathrm{F}_{0}$ was more sensitive to the increasing age of citrus plant than $F_{v} / F_{m}$. There were no significant differences between the leaves of the $30 \mathrm{~s}$ and $8 \mathrm{~s}$ in any of the Chl fluorescence 


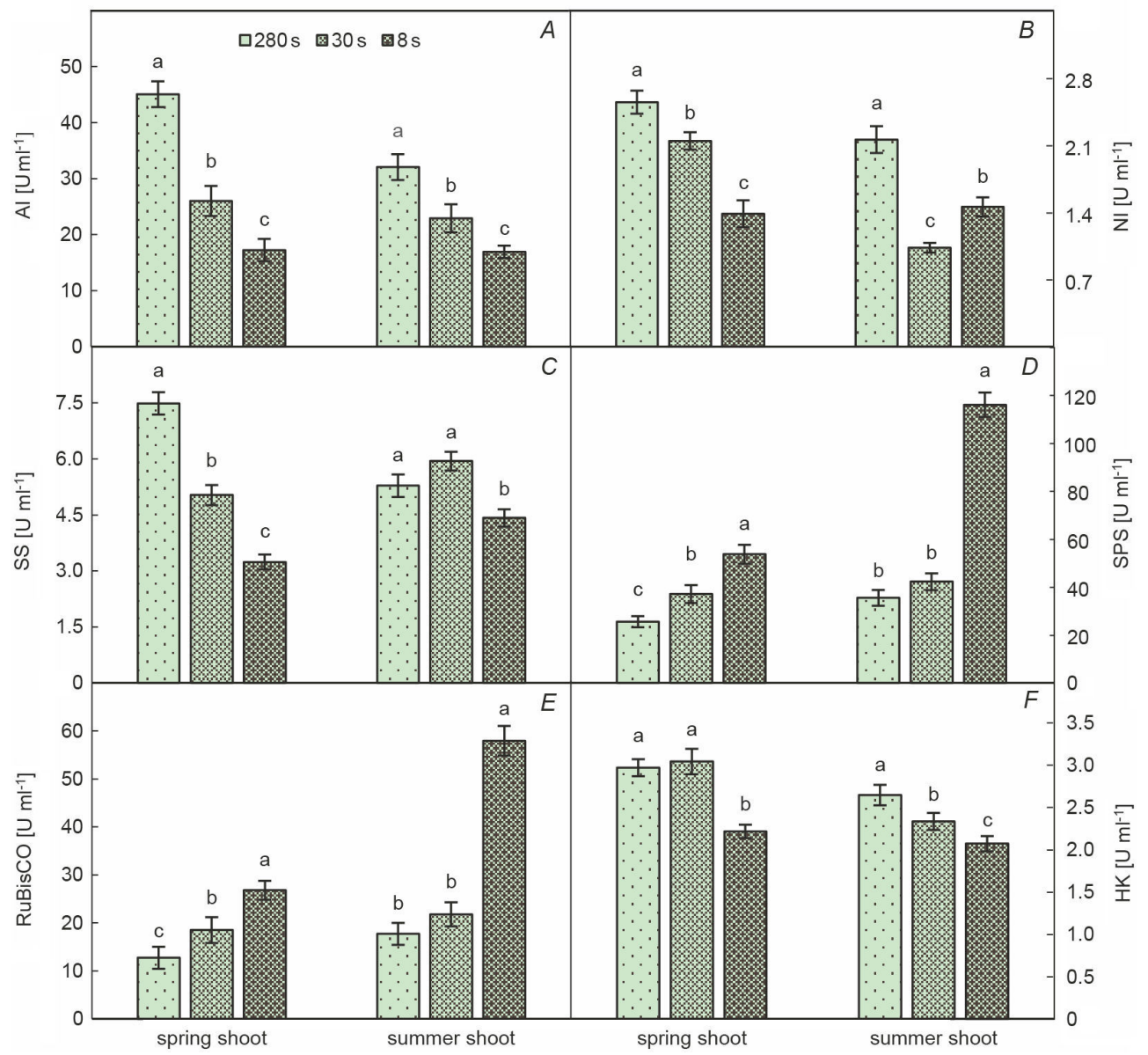

Fig. 4. Photosynthesis-related enzyme activities in spring and summer shoots of 'Huangguogan' trees of different ages. $(A)$ acidic invertase, AI. $(B)$ neutral invertase, NI. (C) sucrose synthetase, SS. $(D)$ sucrose phosphate synthase, SPS. (E) ribulose-1,5-bisphosphate carboxylase/oxygenase, Rubisco. $(F)$ hexokinase, HK. Different letters above the bars indicate significant differences at the $5 \%$ probability level.

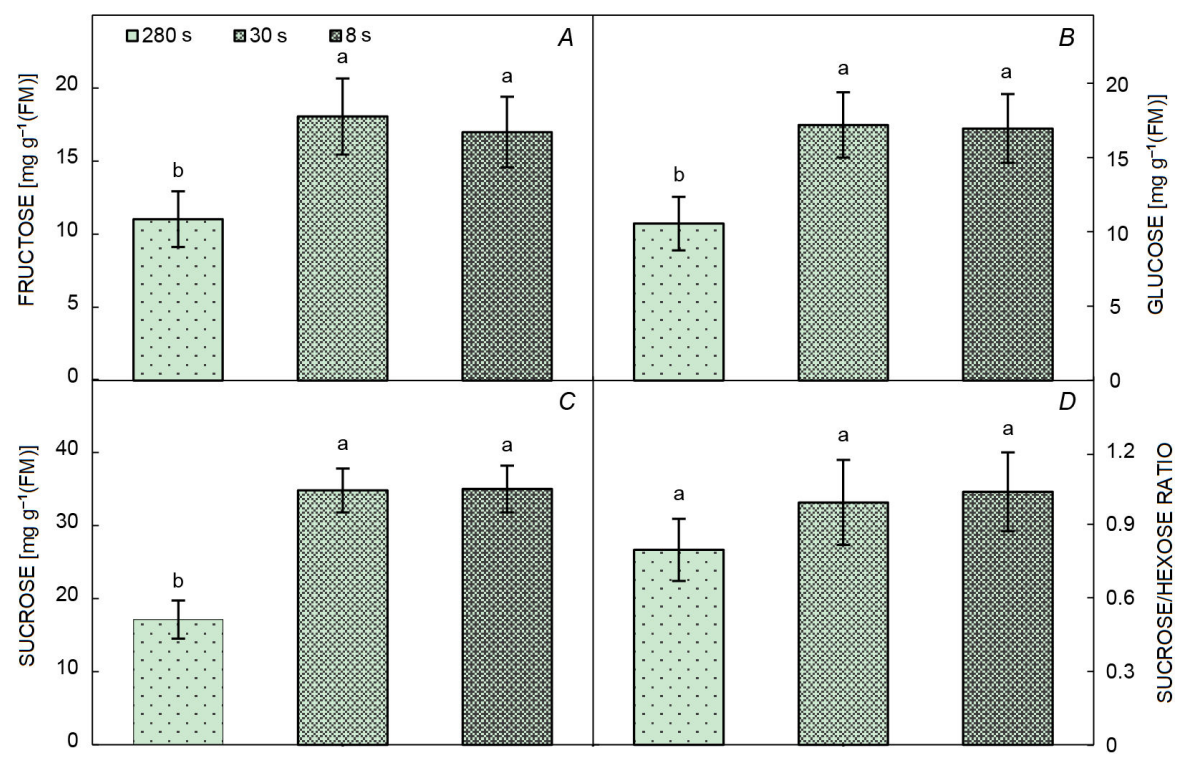

Fig. 5. Fructose $(A)$, glucose $(B)$, and sucrose $(C)$ contents and sucrose/hexose ratio $(D)$ in the fruits of 'Huangguogan' of different ages. Different letters above the bars indicate significant differences at the $5 \%$ probability level. 
parameters in the spring and summer shoots (Fig. 2). This suggests that the photochemical activity of leaves was inhibited to a certain extent by older tree age. However, 'Huangguogan' has relatively high photochemical activity compared to that of other trees, which may have caused the photosynthetic carbon assimilation rate maintained at a relatively high level, especially in the summer shoots. Our analysis of indices related to photosynthesis showed that the leaves of the $8 \mathrm{~s}$ and $30 \mathrm{~s}$ exhibited higher $\mathrm{q}_{\mathrm{p}}$ and $\mathrm{F}_{\mathrm{v}}{ }^{\prime} / \mathrm{F}_{\mathrm{m}}{ }^{\prime}$, but lower $\mathrm{F}_{0}, \mathrm{~F}_{\mathrm{v}} / \mathrm{F}_{\mathrm{m}}$, and NPQ compared to those of the 280 s.

Based on these results, when compared to 280 s, the $8 \mathrm{~s}$ and $30 \mathrm{~s}$ were more effective in utilizing chlorophyll and activating $\mathrm{q}_{\mathrm{P}}$, but less effective in utilizing NPQ, as suggested in previous studies (Allahverdiyeva et al. 2005, Aldea et al. 2006, Murchie and Lawson 2013). The $\mathrm{q}_{\mathrm{P}}$ reflects the share of the absorption of light energy by PSII antenna pigment molecules for photochemical electron transfer. NPQ reflects the proportion of energy absorbed by the PSII reaction center that cannot be used for photosynthetic electron transport, but is dissipated in the form of heat energy (Zhang et al. 2011a). We found that $\mathrm{q}_{\mathrm{P}}$ was the lowest in the leaves of spring shoots of the 280s, and NPQ slightly increased in these leaves. NPQ can dissipate excess light energy in the form of heat energy, thereby protecting photosynthetic organs from light damage (Chen et al. 1995).

As reported by other researchers, sugars, acidity, and aroma are all closely related to fruit quality; overall fruit quality is determined by the total sugar content and composition (Keutgen and Pawelzik 2008). Sucrose is the main component of sweetness, and is transported through sieve elements, and then directly enters into the sink organs through the plasmodesmata or apoplastic space (Zhang et al. 2011b, Pressman et al. 2012). Sucrose and its main components, glucose and fructose, are some of the most important sugars for the process of carbon assimilation, during which these sugars are transported into sink tissues, such as fruits, shoots, and other tissues (Itai and Tanahashi 2008). In the present study, the fructose and glucose contents of fruits from the 30 s and 8 s were nearly equal and were the primary sugars in the ripe fruits of these trees. The fructose and glucose contents of the $30 \mathrm{~s}$ and $8 \mathrm{~s}$ were also clearly different from those in fruits from the 280 s. The differences in fructose and glucose contents, and the sucrose/hexose ratio were associated with changes in AI, NI, SPS, SS, Rubisco, and HK activities. As reported previously, these results suggest that $\mathrm{A} / \mathrm{N}-\mathrm{INV}$ plays an important role in sucrose metabolism (Dahro et al. 2016). In addition, NI may increase a fruit sink strength by increasing hexose concentrations (Zhang et al. 2012). In plant tissues, SS activity is related to the direction of reversible sucrose cleavage, it has a high demand for carbohydrate biosynthesis and respiratory pathways as well as the synthesis of cellulose and callose (Koch 2004). SS plays a key role in the process of sucrose breaking into UDP-glucose and the possible conservation of energy through activation of the hexose moiety by UDP (Verma et al. 2011). In many species, SS plays a very important part in regulating sink strength (Carlson et al. 2002). In the current study, fruits of 8- and 30-year-old trees actively accumulated the end products of photosynthesis, which was verified by the higher $P_{\mathrm{N}}$ of leaves in the spring shoots, more stable Chl fluorescence imaging, lower AI and NI activities, and higher SPS activity relative to the fruits of 280-year-old trees.

The present results show that $P_{\mathrm{N}}$ was positively and significantly correlated with $g_{\mathrm{s}}, C_{\mathrm{i}}$, and $E$, but was negatively correlated with $\mathrm{F}_{\mathrm{v}} / \mathrm{F}_{\mathrm{m}}$ (Fig. 3). This indicated that, with increasing tree age, stomatal limitation became increasingly important limiting factor for $\mathrm{CO}_{2}$ assimilation. This result coincides with those of Hou et al. (2014). The linear relationship between $P_{\mathrm{N}}$ and $\Phi_{\mathrm{PSII}}$ was negative, and the linear relationship between $P_{\mathrm{N}}$ and ETR was positive (Fig. $3 E, F$ ). Although the $\Phi_{\mathrm{PSII}}$ was negatively correlated with $P_{\mathrm{N}}$, there was no significant difference in the $\Phi_{\text {PSII }}$ at different tree ages. All the results proved that stomatal closure and altered Rubisco activity were probably the main limiting factor for $P_{\mathrm{N}}$ with the increasing of Huangguogan plant age.

Conclusions: This study investigated the effects of tree age on photosynthesis, chlorophyll fluorescence, photosynthesis-related enzymes, and fruit sugar content in Citrus 'Huangguogan'. With increasing tree age, there was some inhibition of photosynthesis related to the decrease in $P_{\mathrm{N}}$ caused by changes in $g_{\mathrm{s}}, C_{\mathrm{i}}, E$, and chlorophyll fluorescence. In addition, this study revealed that the photochemical activity of leaves was inhibited to a certain extent by the old age. However, the relatively high photochemical activity of 'Huangguogan' may cause the photosynthetic carbon assimilation rate to be maintained at a relatively high level, especially in the summer shoots. Finally, stomatal limitation was a limiting factor for carbon assimilation in the older trees. The differences observed in fructose and glucose contents and the sucrose/ hexose ratio were accompanied by dramatic differences in AI, NI, SPS, SS, Rubisco, and HK activities. Our results provide some preliminary data for elucidating the physiological and biochemical effects of age on citrus. Therefore, the molecular mechanisms by which tree age affects photosynthesis in citrus should be investigated in the future.

\section{References}

Aldea M., Frank T.D., DeLucia E.H.: A method for quantitative analysis of spatially variable physiological processes across leaf surfaces. - Photosynth. Res. 90: 161-172, 2006.

Allahverdiyeva Y., Mamedov F., Mäenpää P. et al.: Modulation of photosynthetic electron transport in the absence of terminal electron acceptors: Characterization of the $r b c L$ deletion mutant of tobacco. - BBA-Bioenergetics 1709: 69-83, 2005.

Balibrea M.E., Cuartero J., Bolarín M.C., Pérez-Alfocea F.: Sucrolytic activities during fruit development of Lycopersicon genotypes differing in tolerance to salinity. - Physiol. Plantarum 118: 38-46, 2003.

Basson C.E., Groenewald J.-H., Kossmann J. et al.: Sugar and acid-related quality attributes and enzyme activities in strawberry fruits: Invertase is the main sucrose hydrolysing enzyme. - Food Chem. 121: 1156-1162, 2010.

Biswas M.K., Chai L., Amar M.H. et al.: Comparative analysis of 
genetic diversity in Citrus germplasm collection using AFLP, SSAP, SAMPL and SSR markers. - Sci. Hortic.-Amsterdam 129: 798-803, 2011.

Carlson S.J., Chourey P.S., Helentjaris T., Datta R.: Gene expression studies on developing kernels of maize sucrose synthase (SuSy) mutants show evidence for a third SuSy gene. - Plant Mol. Biol. 49: 15-29, 2002.

Chen Y.Z., Li X.P., Xia L. et al.: [The application of chlorophyll fluorescence technique in the study of responses of plant to environmental stresses.] - J. Trop. Subtrop. Bot. 3: 79-86, 1995. [In Chinese]

Dahro B., Wang F., Peng T., Liu J.H.: PtrA/NINV, an alkaline/ neutral invertase gene of Poncirus trifoliata, confers enhanced tolerance to multiple abiotic stresses by modulating ROS levels and maintaining photosynthetic efficiency. - BMC Plant Biol. 16: 76, 2016.

Dai Z., Wu H., Baldazzi V. et al:: Inter-species comparative analysis of components of soluble sugar concentration in fleshy fruits. - Front. Plant Sci. 7: 649, 2016.

Deng S., Mai Y., Niu J.: Fruit characteristics, soluble sugar compositions and transcriptome analysis during the development of Citrus maxima "seedless", and identification of SUS and INV genes involved in sucrose degradation. - Gene 689: 131-140, 2019.

Dong T., Xiong B., Huang S. et al.: Investigation of the cause of reduced sugar content in Kiyomi tangor fruit of Ziyang xiangcheng (Citrus junos Sieb. ex Tanaka) rootstock. - Sci. Rep.-UK 9: 19263, 2019.

Foyer C.H., Shigeoka S.: Understanding oxidative stress and antioxidant functions to enhance photosynthesis. - Plant Physiol. 155: 93-100, 2011.

Gao M., Qi Y., Song W., Xu H.: Effects of di-n-butyl phthalate and di (2-ethylhexyl) phthalate on the growth, photosynthesis, and chlorophyll fluorescence of wheat seedlings. - Chemosphere 151: 76-83, 2016.

Gibson S.I.: Control of plant development and gene expression by sugar signaling. - Curr. Opin. Plant Biol. 8: 93-102, 2005.

Giese J.O., Herbers K., Hoffmann M. et al.: Isolation and functional characterization of a novel plastidic hexokinase from Nicotiana tabacum. - FEBS Lett. 579: 827-831, 2005.

Guidi L., Mori S., Degl'Innocenti E., Pecchia S.: Effects of ozone exposure or fungal pathogen on white lupin leaves as determined by imaging of chlorophyll $a$ fluorescence. - Plant Physiol. Bioch. 45: 851-857, 2007.

Guo J., Jermyn W.A., Turnbull M.H.: Carbon assimilation, partitioning and export in mature cladophylls of two asparagus (Asparagus officinalis) cultivars with contrasting yield. Physiol. Plantarum 115: 362-369, 2002.

Gupta A.K., Kaur N.: Sugar signalling and gene expression in relation to carbohydrate metabolism under abiotic stresses in plants. - J. Biosci. 30: 761-776, 2005.

Hou W., Sun A.H., Yang F.S. et al.: [Effects of low temperature stress on photosynthesis and chlorophyll fluorescence in watermelon seedlings.] - Guangdong Agricultural Sciences 13: 35-39, 2014. [In Chinese]

Huang W.X., Cao Y., Huang L.J. et al.: Differential expression of acid invertase genes in roots of metallicolous and nonmetallicolous populations of Rumex japonicus under copper stress. - Chemosphere 84: 1432-1439, 2011.

Huang Y., Xiong Z.T., Dai L.P., Gao J.Q.: Effect of $\mathrm{Cu}$ stress on the invertase activity and root growth in two populations of Rumex dentatus L. with different $\mathrm{Cu}$ tolerance. - Environ. Toxicol. 23: 443-450, 2008.

Hussain M.I., Reigosa M.J.: A chlorophyll fluorescence analysis of photosynthetic efficiency, quantum yield and photon energy dissipation in PSII antennae of Lactuca sativa L. leaves exposed to cinnamic acid. - Plant Physiol. Bioch. 49: 1290-1298, 2011.

Itai A., Tanahashi T.: Inhibition of sucrose loss during cold storage in Japanese pear (Pyrus pyrifolia Nakai) by 1-MCP. Postharvest Biol. Tec. 48: 355-363, 2008.

Jahan B., AlAjmi M.F., Rehman M.T., Khan N.A.: Treatment of nitric oxide supplemented with nitrogen and sulfur regulates photosynthetic performance and stomatal behavior in mustard under salt stress. - Physiol Plantarum 168: 490-510, 2020.

Keunen E., Peshev D., Vangronsveld J. et al.: Plant sugars are crucial players in the oxidative challenge during abiotic stress: extending the traditional concept. - Plant Cell Environ. 36: $1242-1255,2013$

Keutgen A.J., Pawelzik E.: Quality and nutritional value of strawberry fruit under long term salt stress. - Food Chem. 107: 1413-1420, 2008.

Kitajima M., Butler W.L.: Quenching of chlorophyll fluorescence and primary photochemistry in chloroplasts by dibromothymoquinone. - BBA-Bioenergetics 376: 105-115, 1975.

Koch K.: Sucrose metabolism: regulatory mechanisms and pivotal roles in sugar sensing and plant development. - Curr. Opin. Plant Biol. 7: 235-246, 2004.

Koch K.E.: Carbohydrate-modulated gene expression in plants. Annu. Rev. Plant Phys. 47: 509-540, 1996.

Li Z., Palmer W.M., Martin A.P. et al.: High invertase activity in tomato reproductive organs correlates with enhanced sucrose import into, and heat tolerance of, young fruit. - J. Exp. Bot. 63: 1155-1166, 2012.

Liao L., Cao S.Y., Rong Y., Wang Z.: Effects of grafting on key photosynthetic enzymes and gene expression in the citrus cultivar Huangguogan. - Genet Mol. Res. 15: 15017690, 2016.

Liu Y., Liu Q., Xiong J., Deng X.: Difference of a citrus lateripening mutant (Citrus sinensis) from its parental line in sugar and acid metabolism at the fruit ripening stage. - Sci. China C Life Sci. 50: 511-517, 2007.

Lobo A.K.M., Martins M.O., Lima Neto M.C. et al.: Exogenous sucrose supply changes sugar metabolism and reduces photosynthesis of sugarcane through the down-regulation of Rubisco abundance and activity. - J. Plant Physiol. 179: 113$121,2015$.

Lou Y., Gou J.Y., Xue H.W.: PIP5K9, an Arabidopsis phosphatidylinositol monophosphate kinase, interacts with a cytosolic invertase to negatively regulate sugar-mediated root growth. - Plant Cell 19: 163-181, 2007.

Maestri E., Marmiroli M., Visioli G., Marmiroli N.: Metal tolerance and hyperaccumulation: Costs and trade-offs between traits and environment. - Environ. Exp. Bot. 68: $1-13,2010$.

Marsic N.K., Vodnik D., Mikulic-Petkovsek M. et al.: Photosynthetic traits of plants and the biochemical profile of tomato fruits are influenced by grafting, salinity stress, and growing season. - J. Agr. Food Chem. 66: 5439-5450, 2018.

Martín M.L., Lechner L., Zabaleta E.J., Salerno G.L.: A mitochondrial alkaline/neutral invertase isoform (A/N-InvC) functions in developmental energy-demanding processes in Arabidopsis. - Planta 237: 813-822, 2013.

Mishra P., Dubey R.S.: Effect of aluminium on metabolism of starch and sugars in growing rice seedlings. - Acta Physiol. Plant. 30: 265, 2008.

Murchie E.H., Lawson T.: Chlorophyll fluorescence analysis: a guide to good practice and understanding some new applications. - J. Exp. Bot. 64: 3983-3998, 2013.

Nguyen-Quoc B., Foyer C.H.: A role for 'futile cycles' involving invertase and sucrose synthase in sucrose metabolism of 
tomato fruit. - J. Exp. Bot. 52: 881-889, 2001.

Ohnishi N., Wacera W. F., Sakamoto W.: Photosynthetic responses to high temperature and strong light suggest potential postflowering drought tolerance of sorghum Japanese landrace Takakibi. - Plant Cell Physiol. 60: 2086-2099, 2019.

Patel R.K., Maiti C.S., Deka B.C. et al.: Physical and biological changes in guava (Psidium guajava L.) during various stages of fruit growth and development. - Int. J. Agr. Environ. Biotech. 8: 75, 2015.

Paudel I., Shaviv A., Bernstein N. et al.: Lower leaf gas-exchange and higher photorespiration of treated wastewater irrigated Citrus trees is modulated by soil type and climate. - Physiol. Plantarum 156: 478-496, 2016.

Poorter H., Niklas K.J., Reich P.B. et al.: Biomass allocation to leaves, stems and roots: Meta-analyses of interspecific variation and environmental control. - New Phytol. 193: 3050, 2012.

Pressman E., Shaked R., Shen S. et al:: Variations in carbohydrate content and sucrose-metabolizing enzymes in tomato (Solanum lycopersicum L.) stamen parts during pollen maturation. - Am. J. Plant Sci. 3: 252-260, 2012.

Roitsch T.: Source-sink regulation by sugar and stress. - Curr. Opin. Plant Biol. 2: 198-206, 1999.

Roitsch T., González M.C.: Function and regulation of plant invertases: sweet sensations. - Trends Plant Sci. 9: 606-613, 2004.

Rosa M., Prado C., Podazza G. et al.: Soluble sugars metabolism, sensing and abiotic stress: A complex network in the life of plants. - Plant Signal. Behav. 4: 388-393, 2009.

Ruan Y.L., Jin Y., Yang Y.J. et al.: Sugar input, metabolism, and signaling mediated by invertase: roles in development, yield potential, and response to drought and heat. - Mol. Plant 3: 942-955, 2010.

Sharma D.K., Fernández J.O., Rosenqvist E. et al.: Genotypic response of detached leaves versus intact plants for chlorophyll fluorescence parameters under high temperature stress in wheat. - J. Plant Physiol. 171: 576-586, 2014.

Sicher R.C., Bunce J.A.: Adjustments of net photosynthesis in Solanum tuberosum in response to reciprocal changes in ambient and elevated growth $\mathrm{CO}_{2}$ partial pressures. - Physiol. Plantarum 112: 55-61, 2001.

Song L.L., Zhao H.Q., Zhu X.Q. et al.: [Effects of high temperature stress on photosynthesis and chlorophyll fluorescence of rice.] - J. Anhui Agr. Sci. 39: 13348-13353, 2011. [In Chinese]

Stobrawa K., Lorenc-Plucińska G.: Changes in carbohydrate metabolism in fine roots of the native European black poplar (Populus nigra L.) in a heavy-metal-polluted environment. Sci. Total Environ. 373: 157-165, 2007.

Sturm A., Tang G.Q.: The sucrose-cleaving enzymes of plants are crucial for development, growth and carbon partitioning. Trends Plant Sci. 4: 401-407, 1999.

Tsai Y.C., Chen K.C., Cheng T.S. et al.: Chlorophyll fluorescence analysis in diverse rice varieties reveals the positive correlation between the seedlings salt tolerance and photosynthetic efficiency. - BMC Plant Biol. 19: 403, 2019.

Vargas W.A., Pontis H.G., Salerno G.L.: Differential expression of alkaline and neutral invertases in response to environmental stresses: characterization of an alkaline isoform as a stressresponse enzyme in wheat leaves. - Planta 226: 1535-1545, 2007.

Vargas W.A., Pontis H.G., Salerno G.L.: New insights on sucrose metabolism: evidence for an active $\mathrm{A} / \mathrm{N}-\mathrm{Inv}$ in chloroplasts uncovers a novel component of the intracellular carbon trafficking. - Planta 227: 795-807, 2008.

Verma A.K., Upadhyay S.K., Verma P.C. et al.: Functional analysis of sucrose phosphate synthase (SPS) and sucrose synthase (SS) in sugarcane (Saccharum) cultivars. - Plant Biol. 13: 325-332, 2011.

Wang H.L., Lee P.D., Chen W.L. et al.: Osmotic stress-induced changes of sucrose metabolism in cultured sweet potato cells. - J. Exp. Bot. 51: 1991-1999, 2000.

Xiang L., Le Roy K., Bolouri-Moghaddam M.R. et al.: Exploring the neutral invertase-oxidative stress defence connection in Arabidopsis thaliana. - J. Exp. Bot. 62: 3849-3862, 2011.

Xie D.F., Zhang G.C., Xia X.X. et al.: The effects of phenolic acids on the photosynthetic characteristics and growth of Populus $\times$ euramericana cv. 'Neva' seedlings. Photosynthetica 56: 981-988, 2018.

Xiong B., Ye S., Qiu X. et al.: Exogenous spermidine alleviates fruit granulation in a Citrus cultivar (Huangguogan) through the antioxidant pathway. - Acta Physiol. Plant. 39: 98, 2017.

Xiong Z.T., Wang T., Liu K. et al.: Differential invertase activity and root growth between $\mathrm{Cu}$-tolerant and non-tolerant populations in Kummerowia stipulacea under $\mathrm{Cu}$ stress and nutrient deficiency. - Environ. Exp. Bot. 62: 17-27, 2008.

Ye X., Chen X.F., Deng C.L. et al.: Magnesium-deficiency effects on pigments, photosynthesis and photosynthetic electron transport of leaves, and nutrients of leaf blades and veins in Citrus sinensis seedlings. - Plants-Basel 8: 389, 2019.

Young J.N., Heureux A.M.C., Sharwood R.E. et al.: Large variation in the Rubisco kinetics of diatoms reveals diversity among their carbon-concentrating mechanisms. - J. Exp. Bot. 67: 3445-3456, 2016.

Yousuf P.Y., Abd Allah E.F., Nauman M. et al:: Responsive proteins in wheat cultivars with contrasting nitrogen efficiencies under the combined stress of high temperature and low nitrogen. - Genes-Basel 8: 356, 2017.

Zeeman S.C., Thorneycroft D., Schupp N. et al.: Plastidial $\alpha$-glucan phosphorylase is not required for starch degradation in Arabidopsis leaves but has a role in the tolerance of abiotic stress. - Plant Physiol. 135: 849-858, 2004.

Zhang G.W., Zhang L., Tang M.X. et al.: [Diurnal variation of gas exchange and chlorophyll fluorescence parameters of cotton functional leaves under effects of soil salinity.] - Chin. J. Appl. Ecol. 22: 1771-1781, 2011a. [In Chinese]

Zhang L., Xiong Z.T., Xu Z.R. et al.: Cloning and characterization of acid invertase genes in the roots of the metallophyte Kummerowia stipulacea (Maxim.) Makino from two populations: Differential expression under copper stress. Ecotox. Environ. Safe. 104: 87-95, 2014.

Zhang X.M., Dou M.A., Yao Y.L. et al.: Dynamic analysis of sugar metabolism in different harvest seasons of pineapple (Ananas comosus L.(Merr.)). - Afr. J. Biotechnol. 10: 27162723, 2011b.

Zhang X.M., Wang W., Du L.Q. et al.: Expression patterns, activities and carbohydrate-metabolizing regulation of sucrose phosphate synthase, sucrose synthase and neutral invertase in pineapple fruit during development and ripening. - Int. J. Mol. Sci. 13: 9460-9477, 2012.

Zhou W., Sui Z., Wang J. et al.: Effects of sodium bicarbonate concentration on growth, photosynthesis, and carbonic anhydrase activity of macroalgae Gracilariopsis lemaneiformis, Gracilaria vermiculophylla, and Gracilaria chouae (Gracilariales, Rhodophyta). - Photosynth. Res. 128: 259270, 2016.

(C) The authors. This is an open access article distributed under the terms of the Creative Commons BY-NC-ND Licence. 